\title{
Study of airfoil shape optimization by using the evolutionary method
}

\author{
Oranit Traisak ${ }^{1, *}$, Teetut Dolwichai ${ }^{1}$, Jiraphon Srisertpol ${ }^{1}$ \\ and Chalothorn Thumthae ${ }^{1}$ \\ ${ }^{1}$ School of mechanical engineering, Suranaree University of Technology, Nakhon Ratchasima, \\ Thailand
}

*ORANIT_TRAISAK@hotmail.com

Keywords: Airfoil shape, Evolutionary method, Lift to drag ratio.

\begin{abstract}
Nowadays, Airfoil shape optimization are developed by many researchers. Especially, evolutionary method is taken to find out the shape of airfoil. The main objective work is finding the shape of airfoil that get the aerodynamic properties which good for lift and drag of the aircraft wing. PSO is the evolutionary method that used for this work. The work are combination between the CFD analysis as Reynolds number 550,000 and optimization technique to get the airfoil shape that maximum lift to drag ratio. The result is compared with the standard NACA 2412 airfoil. The optimization airfoil has improved lift to drag ratio compared to the standard NACA 2412 airfoil for $53.22 \%$.
\end{abstract}

\section{Introduction}

The flying is wonderful thing for the human in the past, but nowadays, Aircraft wing is important part which generate the lift force for lift the aircraft up, at the same time, Thrust from aircraft engine induce the drag force which resist forward movement of aircraft. The vertical movement of aircraft can be occurred by the magnitude of lift are greater than its weight. The forward movement can be occurred by the magnitude of thrust are greater than the drag force.

Airfoil is the cross-section of aircraft wing. The shape optimization of the airfoil has 2 methods: First is called as Inverse Design (ID), It's target specification then created airfoil for successful. And second is called as Direct Numerical Optimization (DNO), It's geometry creative for improve aerodynamic performance. [1] There are many researchers to find out the optimum its shape under the lift force only. [2] The couple of lift and drag forces for used to optimization the shape has not presented. There are two points of the objective, maximum value of lift and minimum value of drag, so the problem become to bi-objective optimization problem. [6] The combination of both force can be called as lift to drag ratio (L/D). Therefore, the problem will be the single objective which is maximize the $\mathrm{L} / \mathrm{D}$.

Particle Swarm Optimization (PSO) is one of evolutionary method that popular for non-derivative optimization problems, understand easily and simple step when compared with another method. [3] The work used PSO procedure to combine with CFD analysis which used Xfoil program to find out the $\mathrm{L} / \mathrm{D}$ of the randomized airfoil variables.

\section{Airfoil shape optimization}

Airfoil shape profile. The airfoil profile can be generated by setting the point variables and make it continuously by using the spline interpolation. The NACA2412 airfoil shape is created by using the seven points as shown in the Fig. 1. The four points at the lower and upper part of airfoil are assigned as the seven of design variables. The leading and tailing edge are fixed in the same y direction that equal to zero and $\mathrm{x}$ direction are assigned equal one and zero, respectively. The spline interpolation is 
constructed as the cubic spline which good continuously curve, because of it is the second derivative of the line slope which are drawn between all nodes. Its equation shows as Eq. 1

$$
S_{j}(x)=a_{j}+b_{j}\left(x-x_{j}\right)+c_{j}\left(x-x_{j}\right)^{2}+d_{j}\left(x-x_{j}\right)^{3}
$$

Where; $0 \leq j \leq n-1, S_{j}(x)$ is spline of $\left(x_{j}, x_{j}-1\right), n$ is number of spline line, and The $a_{j}, b_{j}, c_{j}$ and $d_{j}$ are constant coefficient.

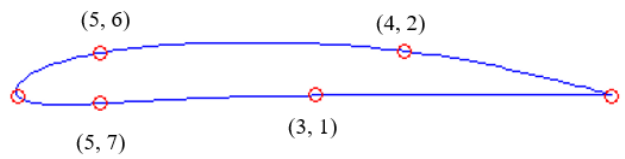

Figure 1. The position of the points to create the airfoil shape.

Aerodynamic parameters. The lift parameter is calculated for the small Angle of Attack (AoA). It can be evaluated the pressure distribution around airfoil by using the panel method. The airfoil is divided as the lines chord that is called as the panel. For example for $N$ panels, we must be had $N+1$ points as shown in Fig. 2

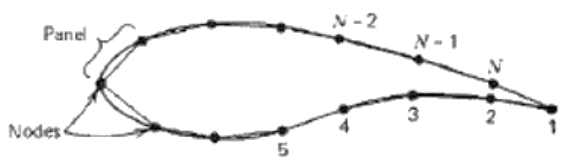

Figure 2. Nodes and panels.

The node number is numbered form the trailing edge forward along the lower airfoil surface to the leading edge and turn back to the upper airfoil surface until reach the starting node. The pressure of each panels are calculated by considering the air flow around the midpoint of individual panel [3]. The pressure coefficient $C_{p}$ for each panel is calculated by Eq. 2, it shows that the pressure distribution along the surface of the airfoil. In addition, it can be used to computed the lift coefficient $\mathrm{C}_{\mathrm{L}}$ by integrating the trapezoidal area pressure distribution.

$$
C_{p}\left(x_{i}, y_{i}\right)=1-\left(V_{t}^{2} / V_{\infty}^{2}\right)
$$

Where $V_{t}$ is tangential velocity

$V_{\infty}$ is free-stream velocity

Drag coefficient can be calculated by using Eq. 3

$$
C_{D}=\frac{C_{f}}{2} U_{s}+C_{\tau}\left(1-U_{s}\right)
$$

Where

$$
C_{f} \text { is skin-friction coefficient }
$$

$C_{\tau}$ is shear stress coefficient

$U_{s}$ is an equivalent normalized wall slip velocity

Lift force $L$ and drag force $D$ can be calculated from Eq. 4 and Eq. 5, respectively.

$$
L=\frac{1}{2} C_{L} \rho V^{2} c
$$




$$
D=\frac{1}{2} C_{D} \rho V^{2} c
$$

So that, the lift to drag ratio $\mathrm{L} / \mathrm{D}$ can be shown as Eq. 6 .

$$
\frac{L}{D}=\frac{C_{L}}{C_{D}}
$$

Particle Swarm Optimization. Particle Swarm Optimization (PSO) is randomlized technique for seek the solution of the optimization problems. It is developed by Dr.Eberhart and Dr.Kennedy in 1995. They are inspired by social behavior of bird flocking or fish schooling which find close to the food. The bird flocking is called as the particle [4,5]. Each particle consists of the velocity and its position as shown in Eq. 7 and Eq. 8, respectively. The food implied as the objective function. For each calculating iteration, the velocity and position can be updated for $i$ particle in individual $k$ iteration

$$
\begin{gathered}
\mathbf{v}_{i}(k)=W \mathbf{v}_{i}(k-1)+C_{1} r_{1}\left(\mathbf{p}_{i}^{\text {best }}-\mathbf{x}_{i}(k-1)\right)+C_{2} r_{2}\left(\mathbf{g}_{i}^{\text {best }}-\mathbf{x}_{i}(k-1)\right) \\
\mathbf{x}_{i}(k)=\mathbf{x}_{i}(k-1)+\mathbf{v}_{i}(k)
\end{gathered}
$$

Where $\quad \mathbf{x}_{i}(k)$ is position of particle at $i$-th and iteration number $k$

$\mathbf{v}_{i}(k) \quad$ is velocity of particle at $i$-th and iteration number $k$

$W \quad$ is inertia weight factor

$C_{1} \quad$ is scaling learning factor cognitive

$C_{2} \quad$ is scaling learning factor social

$\mathbf{p}_{i}^{\text {best }}$ is personal best of each particle

$\mathbf{g}_{i}^{\text {best }}$ is global best of solution in the swarm

The procedure step to find the airfoil shape optimization are shown in Fig. 3.

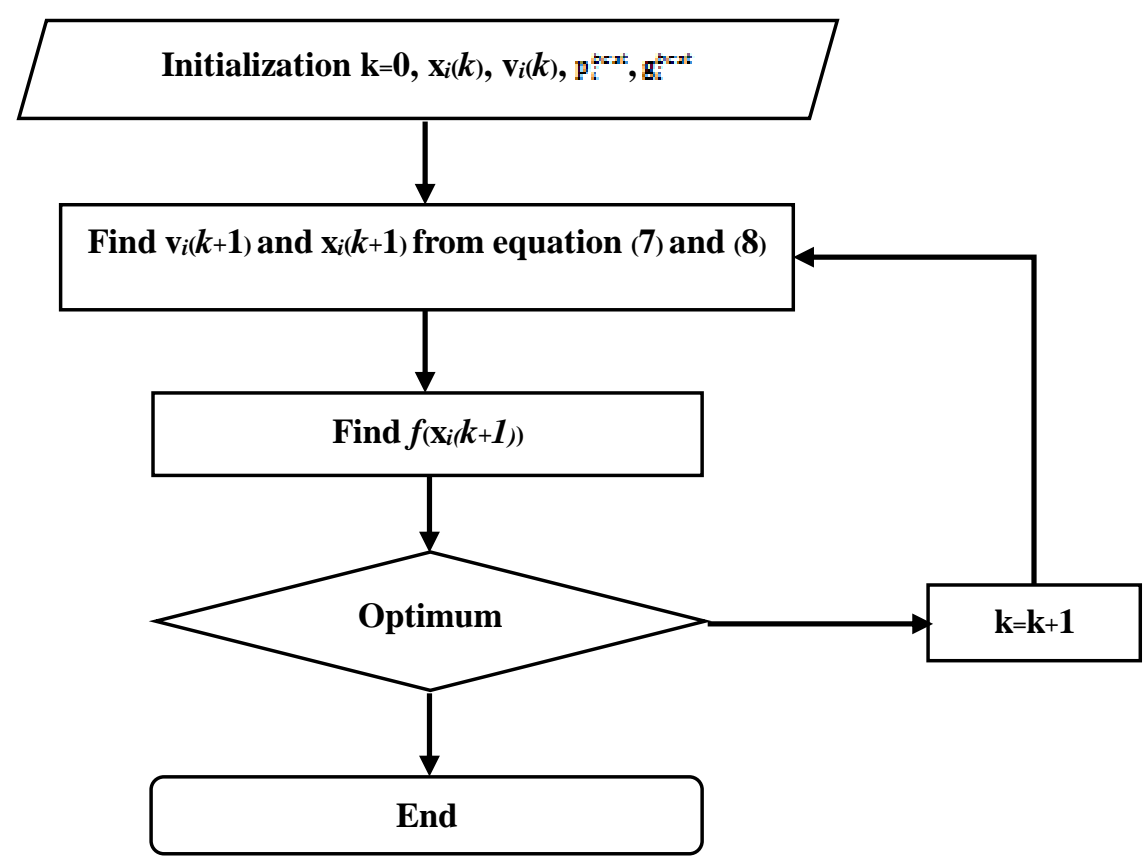

Figure 3. Particle Swarm Optimization precedures.

\section{Optimization procedure}

The optimization works consist of two part, the first is CFD analysis which worked in Xfoil program to find out the L/D, it simulated as subsonic with compressible flow with 550,000 Reynold number. The second is combination the PSO to select the optimum airfoil shape. All of parameters for the two part are shown in Table 1. and Table 2. 
Table 1. CFD analysis parameters

\begin{tabular}{|c|c|}
\hline Angle of attack & 2 degree \\
\hline Flow constrain & $-\quad$ Subsonic and incompressible \\
& $-\quad$ Reynold number 550,000 \\
\hline Geometric & $-\quad$ Max thickness must be less than $12 \%$ chord length \\
& $-\quad$ End of trailing edge is zero. \\
\hline Aerodynamic constrain & Lift to drag ratio not less than original. \\
\hline
\end{tabular}

Table 2. Optimization parameters

\begin{tabular}{|c|l|}
\hline Particle (population) & $10,20,30,40,50,60,70,80,90 \& 100$ \\
\hline Iteration & $10,20,30,40,50,60$ \\
\hline$C_{1} \& C_{2}$ & 2 \\
\hline Objective & Maximum lift to drag ratio (L/D) \\
\hline
\end{tabular}

The combination of the two parts are presented as shown in Fig. 4. It starts from initiation of design the seven of design variable with randomize to create the airfoil. The next step is flow analysis with the Xfoil program to find out the $\mathrm{L} / \mathrm{D}$, after that evaluate optimum airfoil shape by using PSO until we get the best value of $\mathrm{L} / \mathrm{D}$.

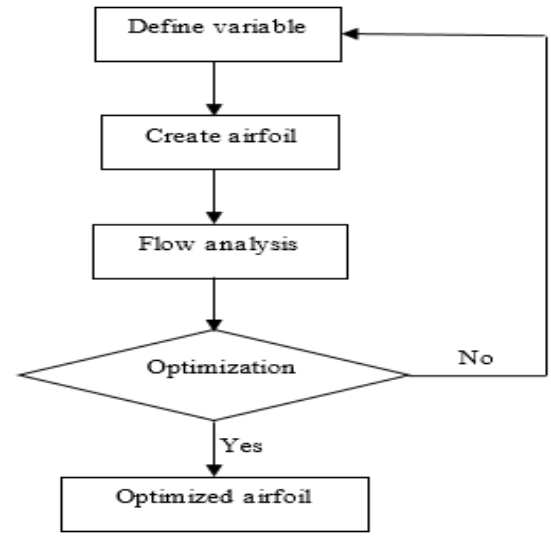

Figure 4. Step to find the optimum airfoil shape.

\section{Result and discussion}

According to the Fig.5, the comparison of the optimized airfoil (blue line) with the standard NACA 2412 airfoil (black line) that is bigger and the trailing edge region has more curved up instant of the same chord length that equal to 1 . These shape effected to the pressure distribution on the lower and upper of the airfoil as shown as Fig. 6(a). and Fig. 6(b). The optimized shape has more under curved area of the pressure distribution than standard airfoil which it effected to lift to drag ratio of greater than too (82.22:54.01). The PSO is used to get the optimized shape with 60 populations and 50 iterations. The modelling with inviscid flow (dashed lines) and viscous flow (continuous line) are slightly not different.

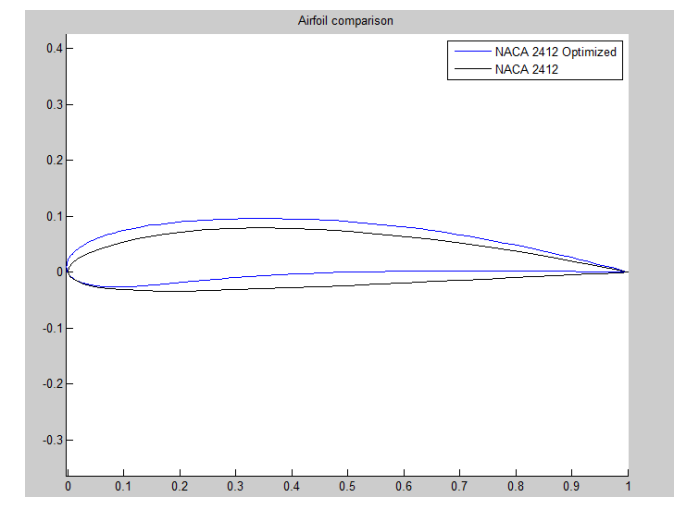

Figure 5. Original NACA 2412 airfoil versus optimized airfoil. 


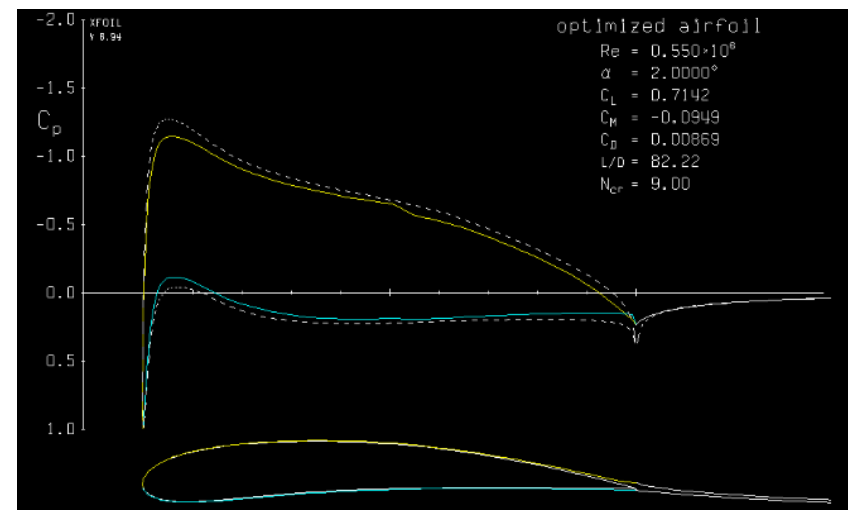

(a)

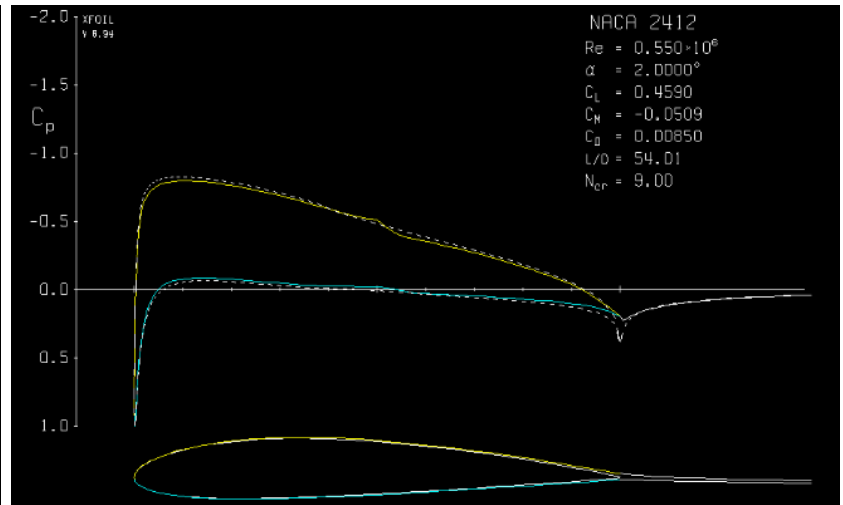

(b)

Figure 6. Pressure distribution and other value of optimized airfoil (a) and original airfoil (b)

\section{Conclusion}

From optimum result found that the optimized airfoil can be made lift to drag ratio greater that the standard airfoil for $52.23 \%$. It shown that can be increased the aircraft gliding capability. The PSO can be used for this work by efficiently.

\section{Acknowledgement}

The author is one research one grant (OROG) scholarship from Suranaree University of Technology.

\section{References}

[1] V. Pierluigi Della, D. Elia, D. Egidio, An airfoil shape optimization technique coupling PARSEC parameterization and evolutionary algorithm, Aerospace Science and Technology 32 (2014) 103-110.

[2] D. Mark, G. Michael B., Viscous-inviscid analysis of transonic and low Reynolds number airfoils, AIAA Journal 25 (1983) 1347-1355.

[3] R. Mukesh, K. Lingadurai, U. Selvakumar, Airfoil shape optimization using non-traditional optimization technique and its validation, Journal of King Saud University - Engineering Sciences 26(2014) 191-197.

[4] B. Sujin, Optimization of mechanical engineering system, first ed., Khon kaen university, Khon Kaen, Thailand, 2013.

[5] Y. Volkan PEHLIVANOĞLU, AY. Serdar, GÜL. Faruk, Improved Particle Swarm Optimization method directed by indirect surrogate modeling, Journal of aeronautics and space technologies $8(2015) 1-10$.

[6] M. Xavier, Automatic 2D Airfoil Generation, Evaluation and Optimisation using Matlab and Xfoil, DTU Mechanical Engineering Section of Fluid Mechanics Technical University of Denmark, 2009. 